\title{
CONVEX BODIES, ECONOMIC CAP COVERINGS, RANIOM POLYTOPES
}

\author{
I. BÁRÁNY AND D. G. LARMAN
}

\$1. Introduction. Let $K$ be a convex compact body with nonempty interior in the $d$-dimensional Euclidean space $R^{d}$ and let $x_{1}, \ldots, x_{n}$ be random points in $K$, independently and uniformly distributed. Define $K_{n}=\operatorname{conv}\left\{x_{1}, \ldots, x_{n}\right\}$. Our main concern in this paper will be the behaviour of the deviation of vol $K_{n}$ from vol $K$ as a function of $n$, more precisely, the expectation of the random variable vol $\left(K \backslash K_{n}\right)$. We denote this expectation by $E(K, n)$.

There are few results known about $E(K, n)$, mainly when $d=2$. (The case $d=1$ is trivial.) Rényi and Sulanke $[18,19]$ proved that for smooth enough convex bodies $K \subset R^{2}$

$$
E(K, n) \approx \text { const }(K) n^{-2 / 3},
$$

where const $(K)$ denotes a constant depending on $K$ only and the notation $f(n) \approx g(n)$ means that $f$ and $g$ are asymptotically equal, i.e., $\lim f(n) / g(n)=1$ when $n \rightarrow \infty$. This has been extended to smooth enough convex bodies $K \subset R^{3}$ by Wieacker [24], who obtained

$$
E(K, n) \approx \text { const }(K) n^{-1 / 2} .
$$

Buchta [4], see also Renyi and Sulanke [18], proved that for a convex polygon $P \subset R^{2}$

$$
E(P, n) \approx \text { const }(P) n^{-1} \log n \text {. }
$$

Little is known in $R^{d}$. Wieacker [24] determined $E\left(B^{d}, n\right)$ where $B^{d}$ denotes the unit ball of $R^{d}$, obtaining

$$
E\left(B^{d}, n\right) \approx \text { const }(d) n^{-2 /(d+1)} .
$$

Groemer [11] proved that for a convex compact body $K \subset R^{d}$ with $\operatorname{vol} K=\operatorname{vol} B^{d}$

$$
E(K, n) \leqslant E\left(B^{d}, n\right),
$$

with equality, if, and only if, $K$ is an ellipsoid.

Until quite recently, nothing has been known about the case of general $d$-polytopes when $d>2$. Buchta [5] proved $E(T, n) \approx \frac{3}{4} n^{-1}(\log n)^{2}$ where $T$ denotes the three dimensional simplex. Dwyer, Kannan and Lovász [14] proved that

$$
E(P, n) \leqslant \text { const }(P) n^{-1}(\log n)^{d}
$$

for a polytope $P \subset R^{d}$. This was improved later by Dwyer [8] to

$$
E(P, n) \leqslant \text { const }(P) n^{-1}(\log n)^{d-1} \text {. }
$$

Dr. Bárány was on leave from the Mathematical Institute of the Hungarian Academy of Sciences, and was supported at University College London by a research fellowship from the Science and Engineering Research Council, U.K., when this paper was written. 
They also proved that for a polytope $P$ having a simple veriex (i.e., a vertex where exactly $d$ facets meet)

$$
E(P, n) \geqslant \text { const }(P) n^{-1}(\log n)^{d-1} .
$$

For further information on the expectation of the number of vertices, surface area, mean width, etc. of $K_{n}$ we refer the reader to Buchta [5], Dwyer [8], Gruber [12], Schneider [22].

We are going to relate $E(K, n)$ to another quantity which we now describe. First, define a map $v: K \rightarrow R$ as

$$
v(x)=\min \{\operatorname{vol}(K \cap H): x \in H, H \text { a halfspace }\} .
$$

Next, for $\varepsilon>0$ define

$$
K(v \leqslant \varepsilon)=\{x \in K: v(x) \leqslant \varepsilon\} .
$$

Sometimes we will write $K(\varepsilon)$ as a shorthand for $K(v \leqslant \varepsilon)$. Here our main result is

THEOREM 1. Assume $K$ is a convex compact body in $R^{d}$ with vol $K=1$. Then, for $n \geqslant n_{0}(d)$ we have

$$
\text { const vol } K(1 / n) \leqslant E(K, n) \leqslant \text { const }(d) \operatorname{vol} K(1 / n) \text {. }
$$

Theorem 1 means that $E(K, n)$ is of the same order of magnitude as vol $K(1 / n)$. We will write this as vol $K(1 / n) \sim E(K, n)$ so the notation $f(n) \sim g(n)$ means that $\lim \inf f(n) / g(n)>0$ and $\lim \inf g(n) / f(n)>0$. This notation implies two constants that are independent of $n$. We mention that in Theorem 1 the constants are independent of $K$ as well. Actually, one of them is universal and the other depends on $d$ only.

Theorem 1 can be used to determine the order of magnitude of $E(K, n)$ for different classes of convex bodies in $R^{d}$. First we prove a general upper bound for vol $K(1 / n) \sim E(K, n)$.

THEOREM 2. Let $K \subset R^{d}$ be a convex compact body with vol $K=1$ and let $\varepsilon>0$. Then

$$
\text { const }(d) \varepsilon(\log (1 / \varepsilon))^{d-1} \leqslant \operatorname{vol} K(\varepsilon)
$$

This theorem is best possible (apart from the constant) as shown by the polytopes.

TheOrem 3. Let $P \subset R^{d}$ be a polytope with vol $P=1$ and let $\varepsilon \geqslant 0$. Then

$$
\text { vol } P(\varepsilon) \leqslant \text { const }(P) \varepsilon(\log (1 / \varepsilon))^{d-1} \text {. }
$$

Theorem 2 and 3 show that vol $P(\varepsilon) \sim \varepsilon(\log (1 / \varepsilon))^{d-1}$ with the implied constant depending on $P$. This, together with Theorem 1 proves that for the class of polytopes $E(P, n) \sim n^{-1}(\log n)^{d-1}$. This result has been obtained independently by Dwyer [8]. The other extreme class of convex bodies is that of the smooth ones. We state an asymptotic result for this class without proof (see Leichweiss [26]). 
THEOREM. For a $\mathscr{C}^{3}$ convex body $K \subset R^{d}$ with vol $K>0$ and positive Gaussian curvature $\kappa$ and for $\varepsilon>0$ we have

$$
\operatorname{vol} K(\varepsilon) \approx \text { const }(d)\left(\int_{\delta K} \kappa^{1 /(d+1)} d S\right) \varepsilon^{2 /(d+1)},
$$

where the integration is taken on the boundary, $\delta K$, of $K$.

This theorem was also proved by Buchta, Gruber, Müller [6]. They noticed that the right-hand side here is a constant multiple of the affine surface area of $K$ ( $c f$. Blaschke [3]) and so Blaschke's affine isoperimetric inequality implies that among all $\mathscr{C}^{3}$ convex bodies of unit volume vol $K(\varepsilon)$ is the largest for the ellipsoids.

Theorem 1, the Theorem above and Groemer's result (1.5) show that for a $\mathscr{C}^{3}$ convex body $K \subset R^{d}, E(K, n) \sim n^{-2 /(d+1)}$ with the implied constants depending on $K$. We are going to prove a theorem that will also yield this. Some preparations are needed. We write $B(\rho, x)$ for the ball of radius $\rho$ and with centre $x \in R^{d}$. Let $p$ be a point on the boundary $\delta K$ of the convex compact set $K \subset R^{d}$. Assume there is a unique outer normal $a$ (with $|a|=1$ ) to $K$ at $p$. Then we call the point $p \rho$-circular if $\rho>0$ and

$$
K \subset B(\rho, p-\rho a) \text {. }
$$

The set of points that are $\rho$-circular for some $\rho>0$ are called circular.

THEOREM 4. If the set of circular points of the boundary of $K$ has positive $(d-1)$-dimensional measure in $\delta K$, then

$$
\operatorname{vol} K(\varepsilon) \geqslant \text { const }(K) \varepsilon^{2 /(d+1)} \text {. }
$$

It is clear that for smooth enough $\left(\mathscr{C}^{3}\right.$, say) convex bodies the conditions of Theorem 4 are satisfied. Thus for smooth convex bodies $K$ we have from Theorem 1, 4 and (1.5) that $E(K, n) \sim n^{-2 /(d+1)}$.

What happens between these two extreme classes is not a mystery: it is the usual unpredictable behaviour. Using (1.5), (1.10) and a general theorem of Gruber [13] (see Schneider [22] for a similar application) one can show this.

Theorem 5. Assume $\omega(n) \rightarrow 0$ and $\Omega(n) \rightarrow \infty$ as $n \rightarrow \infty$. Then for most (in the Baire-category sense) convex bodies $K \subset R^{d}$ with vol $K=1$ one has, for infinitely many $n$

$$
E(K, n)<n^{-1}(\log n)^{d-1} \Omega(n),
$$

and also, for infinitely many $n$

$$
E(K, n)>n^{-2 /(d+1)} \omega(n) .
$$

\$2. Economic cap coverings. One of the main tools in proving Theorem 1 will be the construction of an economic cap covering of $K(v \leqslant \varepsilon)$. For a closed halfpsace $H$ the set $K \cap H$ is called a cap of $K$ whenever it is nonempty. 
THEOREM 6. Assume a convex body $K \subset R^{d}$ is given with vol $K>0$. Take $\varepsilon$ with $0<\varepsilon<\varepsilon_{0}(d)=(2 d)^{-2 d}$. Then there are caps $K_{1}, \ldots, K_{m}$ of $K$ and pairwise disjoint subsets $K_{1}^{\prime}, \ldots, K_{m}^{\prime}, K_{i}^{\prime} \subset K_{i}(i=1, \ldots, m)$ such that

(i) $\bigcup_{1}^{m} K_{i}^{\prime} \subset K(v \leqslant \varepsilon) \subset \bigcup_{1}^{m} K_{i}$,

(ii) $\operatorname{vol} K_{i} \leqslant 6^{d} \varepsilon \quad(i=1, \ldots, m)$,

(iii) $\operatorname{vol} K_{i}^{\prime} \geqslant(6 d)^{-d} \varepsilon \quad(i=1, \ldots, m)$.

This is what is called economic cap covering in the title. In Ewald, Larman, Rogers [9] there is another economic cap covering theorem for the inner parallel body of $K$ (instead of $K(v \leqslant \varepsilon)$ ). Our proof of Theorem 6 is an adaptation of the one in Ewald, Larman, Rogers [9].

Actually, $K \backslash K(v \leqslant \varepsilon)$ is a certain kind of "inner parallel" body to $K$. One may wonder then if its volume is a convex function of $\varepsilon$ or not, or, what is the same, if vol $K(v \leqslant \varepsilon)$ is a concave function of $\varepsilon$ or not. Maybe the $d$-th root of vol $K(v \leqslant \varepsilon)$ is concave. We do not know the answer to these questions. However we can prove some concavity type property of vol $K(v \leqslant \varepsilon)$ that will be useful.

THEOREM 7. Under the assumptions of Theorem 6 one has, for $\lambda \geqslant 1$,

$$
\text { vol } K(v \leqslant \varepsilon) \geqslant \text { const }(d) \lambda^{-d} \text { vol } K(v \leqslant \lambda \varepsilon) \text {. }
$$

We mention a Heilbronn type consequence of Theorem 6 .

TheOREM 8. Assume $P \subset R^{d}$ is a convex polytope with $n$ vertices and vol $P>0$. Then $P$ has $(d+1)$ vertices $x_{0}, x_{1}, \ldots, x_{d}$ such that

$$
\text { vol }\left(\operatorname{conv}\left\{x_{0}, \ldots, x_{d}\right\}\right) / \text { vol } P \leqslant \text { const }(d) n^{-(d+1) /(d-1)} \text {. }
$$

This is a Heilbronn type result $(c f .[15,20])$ because it says that among $n$ points in convex position in $R^{d}$ there is a simplex with small relative volume. This result is known in the plane in a sharper form, see Rényi, Sulanke [19].

Theorem 8 is related to a theorem of Arnold [1] (when $d=2$ ) and Konyagin, Sevastyanov [16] (when $d>2$ ) which states that for a lattice polytope $P \subset R^{d}$ with $n$ vertices and positive volume one has

$$
\text { const }(d) n^{(d+1) /(d-1)} \leqslant \operatorname{vol} P \text {. }
$$

Theorem 8 can be regarded as an extension of (2.3) to the case of general (non-lattice) polytopes. Actually, (2.2) implies (2.3) if the lattice polytope $P$ has no $d+1$ vertices on a hyperplane because then in the left-hand side of (2.2) the volume of the simplex is at least $1 /(d !)$. In fact, the results of Arnold and Konyagin, Sevastyanov are contained in the results of G. E. Andrews [25].

§3. Notation, definitions, basic properties. A cap $C$ of $K$ is a set $C=K \cap H$ where $H$ is a closed halfspace and $K \cap H$ is nonempty. Then $H=\left\{x \in R^{d}: a . x \leqslant \alpha\right\}$ for some $a \in R^{d}$ with $|a|=1$ and $\alpha \in R^{1}$. Here $a . x$ denotes the scalar product of $a$ and $x \in R^{d}$. It will be convenient to write $H=H(a, t)$ with $t=h(a)-\alpha$ where $h(a)=\max \{a \cdot x: x \in K\}$ is the support 
function of $K$. With this notation $t$ is the width of the cap $C$ in direction $a$. In the same spirit we write $H\left(a, t_{1}, t_{2}\right)$ for the strip between the hyperplanes $H\left(a, t_{1}\right)$ and $H\left(a, t_{2}\right)$.

For a cap $C=K \cap H(a, t)$ a point $z \in C$ is called the centre of $C$ if $a . z=h(a)$. A cap may have several centres but we think of a cap as having a fixed centre, say, the centre of gravity of all centres.

For a cap $C=K \cap H(a, t)$ with centre $z$ we define (when $\lambda \geqslant 0$ )

$$
C^{\lambda}=z+\lambda(C-z) \text {. }
$$

Obviously $C^{1}=C$. It is easy to see that for $\lambda \geqslant 1$ one has

$$
C^{\lambda} \supset K \cap H(a, \lambda t) .
$$

When $x \in K$, a minimal cap is defined as a cap $C(x)$ with $x \in C(x)$ and

$$
\text { vol } C(x)=v(x)=\min \{\text { vol } H \cap K: x \in H \text { a halfspace }\} \text {. }
$$

Let us write $H(a=t)$ for the bounding hyperplane of the halfspace $H(a, t)$. A standard variational argument shows that for a minimal cap

$$
C(x)=K \cap H(a, t)
$$

the point $x$ is the centre of gravity of the section $K \cap H(a=t)$.

For $x \in K$ and $\lambda>0$ we call the set

$$
M(x, \lambda)=M_{K}(x, \lambda)=x+\lambda\{(K-x) \cap(x-K)\}
$$

a Macbeath region. Such regions were studied by A. M. Macbeath [17] and Ewald, Larman, Rogers [9]. A Macbeath region is obviously convex and centrally symmetric with centre $x$. We will write $M(x)=M_{K}(x)=M(x, 1)$ when convenient. Define a map $u: K \rightarrow R$ as

$$
u(x)=\operatorname{vol} M(x) .
$$

Macbeath [17] has shown that the set $K(u \geqslant \varepsilon)=\{x \in K: u(x) \geqslant \varepsilon\}$ is convex. The convexity of the set $K(v \geqslant \varepsilon)$ is trivial because it is the intersection of closed halfspaces. It turns out that $K(v \geqslant \varepsilon)$ is "close" to $K(u \geqslant \varepsilon)$.

Theorem 9. Assume $0<\varepsilon<\varepsilon_{1}(d)$. Then

$$
K(v \leqslant \varepsilon) \subset K(u \leqslant 2 \varepsilon) \subset K\left(v \leqslant 2(3 d)^{d} \varepsilon\right)
$$

and

$$
\operatorname{vol} K(v \leqslant \varepsilon) \leqslant \operatorname{vol} K(u \leqslant 2 \varepsilon) \leqslant c_{1}(d) \operatorname{vol} K(v \leqslant \varepsilon)
$$

where $\varepsilon_{1}(d)$ and $c_{1}(d)$ are constants depending on $d$ only.

Here one can take

$$
\varepsilon_{1}(d)=\frac{1}{2}\left(12 d^{3}\right)^{-d} .
$$

We will postpone the proof of this theorem till the last section because we will not use it in the paper.

Denote by $B(r)$ or $B^{d}(r)$ the ball of radius $r$ and centre 0 in $R^{d}$. Throughout the paper we will assume that the given compact convex body $K \subset R^{d}$ (with 
vol $K>0$ ) is in "standard form", i.e.,

$$
B(r) \subset K \subset B(R) \text { and } d r \geqslant R \text {. }
$$

It is well-known (see [7] for instance) that any convex compact body can be transformed by a volume preserving affine transformation into a body $K$ in standard form. Further, it is clear that such a transformation does not change the quantities vol $K(v \leqslant \varepsilon)$, vol $K(u \leqslant \varepsilon)$ or $E(K, n)$ when vol $K=1$.

The assumption vol $K=1$ in the theorems is made for convenience. What we really need is vol $K>0$. At some points we will have to consider sets $K$ with vol $K \neq 1$. Then vol $K(v \leqslant \varepsilon)$ is not affine invariant and it is better to consider instead

$$
\operatorname{vol} K(v \leqslant \varepsilon \operatorname{vol} K) / \operatorname{vol} K
$$

which is affine invariant.

§4. Proof of Theorem 6. We start with two lemmas.

LEMMA $1 . u(x) \leqslant 2 v(x)$.

Proof. Take a halfspace $H$ with $x \in H$. Then

$$
u(x)=\operatorname{vol} M(x) \leqslant 2 \operatorname{vol}(M(x) \cap H) \leqslant 2 \operatorname{vol}(K \cap H),
$$

so

$$
u(x) \leqslant 2 \min \{\operatorname{vol}(K \cap H): x \in H\}=2 v(x) .
$$

LEMMA 2. $v(x) \leqslant(3 d)^{d} u(x)$ if $v(x) \leqslant(2 d)^{-2} d$ or if $u(x) \leqslant\left(12 d^{3}\right)^{-d}$.

Proof. We prove first that $v(x) \leqslant(2 d)^{-2 d}$ implies $v(x) \leqslant(3 d)^{d} u(x)$.

Take a minimal cap $C(x)=K \cap H(a, t)$. As we mentioned earlier $x$ is the centre of gravity of the section $K \cap H(a=t)$. Then, by Lemma 2 of Ewald, Larman, Rogers [9],

$$
C(x) \subset M(x, 3 d)
$$

provided $B(r / 2) \cap H(a, t)$ is empty and $t \leqslant r / 4$.

Assume now that (4.1) fails. Then either $B(r / 2) \cap H(a, t)$ is nonempty or $t \geqslant r / 4$. We show now that both cases contradict the condition $v(x) \leqslant(2 d)^{-2 d}$.

In the first case, i.e., when $B(r / 2) \cap H(a, t) \neq \varnothing$, the set $B(r) \cap H(a, t)$ contains a cap $C_{r}$ of $B(r)$ whose width is $r / 2$. Moreover, by (3.5),

$$
C=K \cap H(a, t) \supset B(r) \cap H(a, t) \supset C_{r}
$$

so vol $C(x) \geqslant \operatorname{vol} C_{r}$. A simple computation shows now that vol $C_{r} \geqslant(2 d)^{-d}$.

In the second case when $t \geqslant r / 4$, i.e., the width of $C(x)$ in direction $a$ is at least $r / 4$, let $z$ be the centre of $C(x)$. Consider the cone $L$ with apex $z$ whose base is the intersection of $B(r)$ with the hyperplane through 0 and orthogonal to $a$. The height of this cone is $h(a) \leqslant R$ and its volume is

$$
\operatorname{vol} L=(1 / d) r^{d-1} \omega_{d-1} h(a)
$$

where $\omega_{d-1}$ is the volume of $B^{d-1}$, the unit ball of $R^{d-1}$. The cap $C(x)$ contains 
the part of this cone $L$ lying in the strip $H(a, 0, t)$. The volume of this part is

$$
\begin{aligned}
\left(\frac{t}{h(a)}\right)^{d} \text { vol } L & =\left(\frac{t}{h(a)}\right)^{d-1} \frac{t}{d} r^{d-1} \omega_{d-1} \geqslant\left(\frac{r}{4 R}\right)^{d-1} \frac{r}{4 d} r^{d-1} \omega_{d-1} \\
& \geqslant\left(\frac{1}{4 d}\right)^{d-1} \frac{1}{4 d} d^{d} r^{d} \omega_{d}\left(\omega_{d-1} / \omega_{d}\right) d^{-d} \\
& \geqslant(4 d)^{-d} R^{d} \omega_{d}\left(\omega_{d-1} / \omega_{d}\right) d^{-d} \geqslant(2 d)^{-2 d}\left(\omega_{d-1} / \omega_{d}\right) \\
& \geqslant(2 d)^{-2 d} .
\end{aligned}
$$

So vol $C(x) \geqslant(2 d)^{-2 d}$. This contradiction shows that (4.1) holds. Then obviously, $v(x) \leqslant(3 d)^{d} u(x)$.

To finish the proof of the lemma we prove now that $u(x) \leqslant\left(12 d^{3}\right)^{-d}$ implies $v(x) \leqslant(2 d)^{-2 d}$. To see this we claim that

$$
K\left(v \geqslant(2 d)^{-2 d}\right) \subset K\left(u \geqslant\left(12 d^{3}\right)^{-d}\right) .
$$

Both sets here are convex (the second by Macbeath's result [17]) and both of them contain the origin. When $x$ is a point on the boundary of $K\left(v \geqslant(2 d)^{-2 d}\right)$, i.e., when $v(x)=(2 d)^{-2 d}$, then by the first part of this proof,

$$
u(x) \geqslant(3 d)^{-d} v(x)=\left(12 d^{3}\right)^{-d}
$$

i.e., $x \in K\left(u \geqslant\left(12 d^{3}\right)^{-d}\right)$.

Now we turn to the proof of Theorem 6 . Consider the set $K(v \geqslant \varepsilon)$ and choose a maximal system of points $x_{1}, x_{2}, \ldots, x_{m}$ from the boundary $\partial K(v \geqslant \varepsilon)$ of the set $K(v \geqslant \varepsilon)$ subject to the condition that

$$
M\left(x_{i}, \frac{1}{2}\right) \cap M\left(x_{j}, \frac{1}{2}\right)=\varnothing \quad \text { when } \quad x_{i} \neq x_{j} .
$$

This maximal system is indeed finite because the sets $M\left(x_{i}, \frac{1}{2}\right)$ are pairwise disjoint, all of them lie in $K$ and

$$
\operatorname{vol} M\left(x_{i}, \frac{1}{2}\right)=2^{-d} \operatorname{vol} M\left(x_{i}, 1\right)=2^{-d} u\left(x_{i}\right) \geqslant(6 d)^{-d} v\left(x_{i}\right)=(6 d)^{-d} \varepsilon
$$

according to Lemma 2 .

Claim 1. $K(v \leqslant \varepsilon) \subset \bigcup\left\{M\left(x_{i}, 5\right): i=1, \ldots, m\right\}$.

Proof. Consider any point $y^{\prime \prime} \in K(v \leqslant \varepsilon)$. As $0 \in$ int $K(v \geqslant \varepsilon)$, the halfline stemming from 0 in direction $y^{\prime \prime}$ intersects the boundary of the convex set $K(v \geqslant \varepsilon)$ and $K$ at the points $y$ and $y^{\prime}$, respectively. Now $x_{1}, \ldots, x_{m}$ form a maximal system in $\delta K(v \geqslant \varepsilon)$ with respect to (4.2) and $y \in \delta K(v \geqslant \varepsilon)$. So there is an $i$ such that

$$
M\left(x_{i}, \frac{1}{2}\right) \cap M\left(y, \frac{1}{2}\right) \neq \varnothing .
$$

Then, by Lemma 1 of Ewald, Larman, Rogers [9],

$$
M(y, 1) \subset M\left(x_{i}, 5\right) .
$$

We will prove now that $y^{\prime} \in M(y, 1)$. This will show that the line segment $\left[y, y^{\prime}\right]$ and, consequently, the point $y^{\prime \prime} \in\left[y, y^{\prime}\right]$ lie in $M(y, 1)$ and this will prove the Claim. 
Assume $y^{\prime} \notin M(y, 1)$. On the line through 0 and $y$ let $z$ be the point at distance $r$ from 0 and such that 0 lies between $z$ and $y$. Then $z \in B(r) \subset K$ and so $y^{\prime} \notin M(y, 1)$ implies

$$
|z-y|<\left|y-y^{\prime}\right|
$$

Consider now the minimal cap $C=C(y)=K \cap H$. Clearly, $H$ cannot contain 0 for otherwise $C$ would contain "half" of the ball $B(r)$ which has volume $\frac{1}{2} r^{d} \omega_{d} \geqslant \frac{1}{2} d^{-d}>\varepsilon=\operatorname{vol} C$. Then $H$ must contain $y^{\prime}$. Then $H$ must contain "half" of the cone $L$ whose apex is $y^{\prime}$ and whose base is the intersection of the set conv $(\{y\} \cup B(r))$ with the halfspace orthogonal to, and passing through, the vector $y$. Computing volumes again

$$
\text { vol } \begin{aligned}
C & \geqslant \frac{1}{2} \text { vol } L \geqslant \frac{1}{2} \frac{1}{d}\left|y^{\prime}\right| r^{d-1} \omega_{d-1}\left(\frac{\left|y-y^{\prime}\right|}{\left|y^{\prime}\right|}\right)^{d} \\
& \geqslant \frac{1}{2 d} r^{d} \omega_{d-1}\left(\frac{|z-y|}{\left|y^{\prime}\right|}\right)^{d} \geqslant \frac{1}{2 d} r^{d} \omega_{d}(r / R)^{d}\left(\omega_{d-1} / \omega_{d}\right)>(2 d)^{-2 d} \geqslant \varepsilon .
\end{aligned}
$$

Now we have an economic cap covering of $K(v \leqslant \varepsilon)$ by Macbeath regions. We are going to turn it into a covering by caps.

For this end consider the minimal cap $C_{i}=C\left(x_{i}\right)=K \cap H\left(a_{i}, t_{i}\right)$, for $i=1, \ldots, m$. Define

$$
\begin{gathered}
K_{i}=K \cap H\left(a_{i}, 6 t_{i}\right), \\
K_{i}^{\prime}=M\left(x_{i}, \frac{1}{2}\right) \cap H\left(a_{i}, t_{i}\right) .
\end{gathered}
$$

We claim that the sets $K_{i}, K_{i}^{\prime}$ satisfy the requirements of the theorem. First, as the sets $M\left(x_{i}, \frac{1}{2}\right)$ are pairwise disjoint, so are the sets $K_{i}^{\prime}$. According to (4.1), $C_{i} \subset M\left(x_{i}, 3 d\right)$ so

$$
\begin{aligned}
\operatorname{vol} K_{i}^{\prime} & =\frac{1}{2} \operatorname{vol} M\left(x_{i}, \frac{1}{2}\right)=\frac{1}{2}(6 d)^{-d} \operatorname{vol} M\left(x_{i}, 3 d\right) \\
& \geqslant \frac{1}{2}(6 d)^{-d} \operatorname{vol} C_{i}=\frac{1}{2}(6 d)^{-d} \varepsilon
\end{aligned}
$$

One can get vol $K_{i}^{\prime} \geqslant(6 d)^{-d} \varepsilon$ from here by observing that the central symmetry of $M\left(x_{i}, 3 d\right)$ and (4.1) imply 2 vol $C_{i} \leqslant \operatorname{vol} M\left(x_{i}, 3 d\right)$.

Notice that $M\left(x_{i}, 1\right)$ lies in the strip $H\left(a_{i}, 0,2 t_{i}\right)$. Then $M\left(x_{i}, 5\right)$ lies in the strip $H\left(a_{i},-4 t_{i}, 6 t_{i}\right)$ as the centre of $M\left(x_{i}, \lambda\right)$ is on the hyperplane $H\left(a_{i}=t_{i}\right)$. Thus

$$
K \cap M\left(x_{i}, 5\right) \subset K \cap H\left(a_{i},-4 t_{i}, 6 t_{i}\right)=K \cap H\left(a_{i}, 6 t_{i}\right)=K_{i}
$$

and indeed

$$
K(v \leqslant \varepsilon) \subset \bigcup_{1}^{m} K_{i}
$$

According to (3.1) and (3.2)

$$
\operatorname{vol} K_{i} \leqslant 6^{d} \operatorname{vol} C_{i}=6^{d} \varepsilon .
$$

Finally, $K_{i}^{\prime} \subset K_{i}$ is evident. 
\$5. Proof of Theorem 7. Let $K_{1}, \ldots, K_{m}$ be the economic cap covering of $K(v \leqslant \varepsilon)$ from Theorem 6 . We will prove that the union of $K_{i}^{d \lambda}$ $(i=1, \ldots, m)$ covers $K(v \leqslant \lambda \varepsilon)$.

So we take a point $x \in K(v \leqslant \lambda \varepsilon)$. We have to show that

$$
x \in K_{1}^{d \lambda} \cup \ldots \cup K_{m}^{d \lambda},
$$

thus we may assume that $x \notin K_{1} \cup \ldots \cup K_{m}$.

The minimal cap $C(x)=K \cap H(a, t)$ has centre $z$ (say), and the line segment $[x, z]$ intersects the boundary of $K(v \leqslant \varepsilon)$ at the point $y$. Clearly $v(y)=\varepsilon$. Let $t^{\prime}$ be the distance of $y$ from the hyperplane $H(a, 0)$ (which supports $K$ at $z)$. Then $y \in H\left(a=t^{\prime}\right)$ and

$$
\begin{aligned}
\varepsilon=v(y) & \leqslant \operatorname{vol}\left(K \cap H\left(a, t^{\prime}\right)\right)=\int_{0}^{t^{\prime}} \operatorname{vol}_{d-1}(K \cap H(a=\tau)) d \tau \\
& \leqslant t^{\prime} \max \left\{\operatorname{vol}_{d-1}(K \cap H(a=\tau)): 0 \leqslant \tau \leqslant t^{\prime}\right\} \\
& \leqslant t^{\prime} \max \left\{\operatorname{vol}_{d-1}(K \cap H(a=\tau)): 0 \leqslant \tau \leqslant t\right\}
\end{aligned}
$$

On the other hand

$$
\lambda \varepsilon \geqslant v(x)=\operatorname{vol} K \cap H(a, t) \geqslant \frac{1}{d} t \max \left\{\operatorname{vol}_{d-1}(K \cap H(a=\tau)): 0 \leqslant \tau \leqslant t\right\},
$$

where the last inequality follows from the fact that the double cone whose base is the maximal section $K \cap H(a=\tau)$ is contained in $C(x)$. Now $t / t^{\prime}=|z-x| /|z-y|$ and so we get

$$
|z-x| \leqslant \lambda d|z-y|
$$

Consider now the cap $K_{i}=K \cap H\left(a_{i}, t_{i}\right)$ from the cap covering that contains $y$. Let $z_{i}$ be the centre of $K_{i}$ and write $y_{i}$ for the intersection $\left[z_{i}, x\right] \cap H\left(a_{i}=t_{i}\right)$. The line $L$ through $z$ and $x$ intersects the hyperplanes $H\left(a_{i}=0\right)$ and $H\left(a_{i}=t_{i}\right)$ at the points $z^{\prime}$ and $y^{\prime}$, respectively. It is easy to check that the points $z^{\prime}, z, y, y^{\prime}, x$ come on $L$ in this order. Then

$$
\frac{\left|x-z_{i}\right|}{\left|y_{i}-z_{i}\right|}=\frac{\left|x-z^{\prime}\right|}{\left|y^{\prime}-z^{\prime}\right|} \leqslant \frac{\left|x-z^{\prime}\right|}{\left|y-z^{\prime}\right|}=\frac{|x-z|+\left|z-z^{\prime}\right|}{|y-z|+\left|z-z^{\prime}\right|} \leqslant \frac{|x-z|}{|y-z|} \leqslant \lambda d \text {. }
$$

So indeed $x \in K_{1}^{\lambda d} \cup \ldots \cup K_{i}^{\lambda d}$. Now

$$
\begin{aligned}
\operatorname{vol} K(v \leqslant \lambda \varepsilon) & \leqslant \sum_{i=1}^{m} \operatorname{vol} K_{i}^{\lambda d} \leqslant(\lambda d)^{d} \sum_{i=1}^{m} \operatorname{vol} K_{i} \leqslant(\lambda d)^{d} 6^{d} m \varepsilon \\
& \leqslant(6 \lambda d)^{d}(6 d)^{d} \sum_{i=1}^{m} \operatorname{vol} K_{i}^{\prime} \leqslant\left(36 \lambda d^{2}\right)^{d} \operatorname{vol} K(v \leqslant \varepsilon)
\end{aligned}
$$

§6. Proof of Theorem 1. To establish the lower bound let $x \in K$ and let $C(x)$ be the corresponding minimal cap. Then

$$
\operatorname{Prob}\left(x \notin K_{n}\right) \geqslant \operatorname{Prob}\left(C(x) \cap K_{n}=\varnothing\right)=(1-v(x))^{n} .
$$


Consequently, for $\varepsilon>0$, we get

$$
\begin{aligned}
E(K, n) & =\int_{K} \operatorname{Prob}\left(x \notin K_{n}\right) \geqslant \int_{K}(1-v(x))^{n} \geqslant \int_{K(v \leqslant \varepsilon)}(1-v(x))^{n} \\
& \geqslant \int_{K(v \leqslant \varepsilon)}(1-\varepsilon)^{n}=(1-\varepsilon)^{n} \text { vol } K(v \leqslant \varepsilon) .
\end{aligned}
$$

Choosing now $\varepsilon=1 / n$ (and assuming $n \geqslant 3$ ) we have

$$
\frac{1}{4} \operatorname{vol} K\left(v \leqslant \frac{1}{n}\right) \leqslant E(K, n) .
$$

Proving the upper bound is more involved. First we use an idea from Bárány and Füredi [2]. Let $x_{1}, \ldots, x_{n}$ be randomly chosen points and write $N(x)=\left\{x_{1}, \ldots, x_{n}\right\} \cap M(x)$ when $x \in K$. Further, denote by $n(x)$ the cardinality of $N(x)$. Now

$$
\begin{aligned}
\operatorname{Prob}\left(x \notin K_{n}\right) & =\sum_{m=0}^{n} \operatorname{Prob}\left(x \notin K_{n} \mid n(x)=m\right) \text { Prob }(n(x)=m) \\
& \leqslant \sum_{m=0}^{n} \operatorname{Prob}(x \notin \operatorname{conv} N(x) \mid n(x)=m) \operatorname{Prob}(n(x)=m) .
\end{aligned}
$$

According to a theorem of Wendel [24] (cf. Füredi [10] as well)

$$
\operatorname{Prob}(x \notin \operatorname{conv} N(x) \mid n(x)=m)=2^{-(m-1)} \sum_{i=1}^{d-1}\left(\begin{array}{c}
m \\
i
\end{array}\right) \text {. }
$$

Using this

$$
\begin{aligned}
\operatorname{Prob}\left(x \notin K_{n}\right) & \leqslant 2 \sum_{m=0}^{n} 2^{-m} \sum_{i=0}^{d-1}\left(\begin{array}{c}
m \\
i
\end{array}\right)[u(x)]^{m}[1-u(x)]^{n-m}\left(\begin{array}{c}
n \\
m
\end{array}\right) \\
& =2 \sum_{i=0}^{d-1} \sum_{m=i}^{n}\left(\begin{array}{c}
m \\
i
\end{array}\right)\left(\begin{array}{c}
n \\
m
\end{array}\right)[u(x) / 2]^{m}[1-u(x)]^{n-m} \\
& =2 \sum_{i=0}^{d-1} \sum_{m=i}^{n}\left(\begin{array}{c}
n \\
i
\end{array}\right)\left(\begin{array}{c}
n-i \\
m-i
\end{array}\right)[u(x) / 2]^{m}[1-u(x)]^{n-m} \\
& =2 \sum_{i=0}^{d-1}\left(\begin{array}{c}
n \\
i
\end{array}\right) \sum_{m=i}^{m}\left(\begin{array}{c}
n-i \\
m-i
\end{array}\right)[u(x) / 2]^{m}[1-u(x)]^{n-m} \\
& =2 \sum_{i=0}^{d-1}\left(\begin{array}{c}
n \\
i
\end{array}\right) \sum_{k=0}^{n-i}\left(\begin{array}{c}
n-i \\
k
\end{array}\right)[u(x) / 2]^{k+i}[1-u(x)]^{n-i-k} \\
& =2 \sum_{i=0}^{d-1}\left(\begin{array}{c}
n \\
i
\end{array}\right)[u(x) / 2]^{i}\left[1-\frac{1}{2} u(x)\right]^{n-i} .
\end{aligned}
$$


Now we integrate

$$
\begin{aligned}
E(K, n) & =\int \operatorname{Prob}\left(x \notin K_{n}\right) d x \\
& \leqslant 2 \sum_{i=0}^{d-1}\left(\begin{array}{l}
n \\
i
\end{array}\right) \int_{K}\left[\frac{1}{2} u(x)\right]^{i}\left[1-\frac{1}{2} u(x)\right]^{n-i} d x \\
& =2 \sum_{i=0}^{d-1}\left(\begin{array}{l}
n \\
i
\end{array}\right) \sum_{\lambda=1}^{n}[(\lambda-1) / n] \leqslant u(x) \leqslant(\lambda / n) \\
& <2 \sum_{i=0}^{d-1}\left(\begin{array}{l}
n \\
i
\end{array}\right) \sum_{\lambda=1}^{n}\left[\frac{1}{2} u(x)\right]^{i}\left[1-\frac{1}{2} u(x)\right]^{n-i} d x \\
& \leqslant 2 \sum_{i=0}^{d-1}\left(\begin{array}{l}
n \\
i
\end{array}\right) \sum_{\lambda=1}^{n}\left(\frac{\lambda}{2 n}\right)^{i}\left(1-\frac{\lambda-1}{2 n}\right)^{n / 2} \operatorname{vol} K\left(u \leqslant \frac{\lambda}{n}\right) .
\end{aligned}
$$

Here

$$
2\left(\begin{array}{c}
n \\
i
\end{array}\right)\left(\frac{\lambda}{n}\right)^{i} \leqslant \lambda^{i} /\left(2^{i} i !\right) \leqslant \lambda^{d-1}
$$

because $i \geqslant d-1$. Moreover, when $n \geqslant 2 d$,

$$
\left(1-\frac{\lambda-1}{2 n}\right)^{n-i} \leqslant\left(1-\frac{\lambda-1}{2 n}\right)^{n-i} \leqslant e^{-(\lambda-1) / 4} \text {. }
$$

According to Lemma 2

$$
K(u \leqslant \lambda / n) \subset K\left(v \leqslant(3 d)^{d} \lambda / n\right)
$$

provided $\lambda / n<\left(12 d^{3}\right)^{-d}$. We set $\Lambda=\left\lfloor\left(12 d^{3}\right)^{-d} n\right\rfloor$ and continue (6.3).

$$
\begin{aligned}
E(K, n) \leqslant & \sum_{\lambda=1}^{\lambda=\Lambda} d \lambda^{d-1} e^{-(\lambda-1) / 4} \text { vol } K\left(v \leqslant(3 d)^{d} \lambda / n\right) \\
& +\sum_{\lambda=\Lambda+1}^{n} d \lambda^{d-1} e^{-(\lambda-1) / 4}
\end{aligned}
$$

as vol $K(v \leqslant \varepsilon) \leqslant \operatorname{vol} K=1$ for every $\varepsilon \geqslant 0$. Here in the first sum

$$
\text { vol } K\left(v \leqslant(3 d)^{d} \lambda / n\right) \leqslant\left[36 \lambda(3 d)^{d} d^{2}\right]^{d} \text { vol } K(v \leqslant 1 / n),
$$

by Theorem 7 , so the first sum is at most

$$
\operatorname{vol} K\left(v \leqslant \frac{1}{n}\right) \sum_{\lambda=1}^{\infty} d \lambda^{d-1} e^{-(\lambda-1) / 4} \lambda^{d}\left[36 d^{2}(3 d)^{d}\right]^{d}<\text { const }(d) \text { vol } K\left(v \leqslant \frac{1}{n}\right) \text {. }
$$

To estimate the second sum in (6.4) observe that it is less than

$$
\sum_{\lambda=1}^{\infty} d \lambda^{d-1} e^{-(\lambda-1) / 8} e^{-\left(12 d^{3}\right)^{-d}(n / 8)}<c_{1}(d) e^{-\left(12 d^{3}\right)^{-d}(n / 8)},
$$

where $c_{1}(d)$ is a constant depending on $d$ only. We need a lower bound on 
CONVEX BODIES, ECONOMIC CAP COVERINGS, RANDOM POLYTOPES

vol $K(v \leqslant 1 / n)$. We could use Theorem 2 , but we prefer the very simple vol $K(v \leqslant \varepsilon) \geqslant \varepsilon$ inequality, which follows from the fact that $C(x) \subset K(v \leqslant \varepsilon)$ for any $x$ with $v(x)=\varepsilon$. Using this the second sum in (6.4) is less than

$$
c_{1}(d) e^{-\left(12 d^{3}\right)^{-d}(n / 8)}<c_{2}(d) \frac{1}{n} \leqslant c_{2}(d) \operatorname{vol} K(v \leqslant 1 / n) .
$$

With (6.5) and (6.6) we get from (6.4) that indeed

$$
E(K, n) \leqslant \text { const }(d) \text { vol } K(v \leqslant 1 / n) .
$$

We mention here that a byproduct of (6.2) is that:

$\operatorname{Prob}\left(x \notin K_{n}\right)$

$\leqslant 2$ Prob (less that $d$ points from $\left\{x_{1}, \ldots, x_{n}\right\}$ lie in $M(x) \cap C(x)$ ).

\$7. Proof of Theorem 2. We start with some notation. Fix $a \in R^{d},|a|=1$ somehow and let $H\left(a=t_{0}\right)$ be the hyperplane whose intersection with $K$ has the largest $(d-1)$-dimensional volume among all hyperplanes $H(a=t)$. Assume the width of $K$ in direction $a$ is at most $2 t_{0}$. If this were not the case we would take $-a$ instead of $a$. As $a$ will be fixed throughout this proof we will write $H(t)=H(a=t)$. Define, further,

$$
Q(t)=H(t) \cap K \quad \text { and } \quad q(t)=\operatorname{vol}_{d-1} Q(t) .
$$

Our choice for $t_{0}$ insures that

$$
\begin{gathered}
q(t) \geqslant\left(t / t_{0}\right)^{d-1} q\left(t_{0}\right) \quad \text { for } 0 \leqslant t \leqslant t_{0} \\
2 t_{0} q\left(t_{0}\right) \geqslant \operatorname{vol} K=1
\end{gathered}
$$

LEMMA 3. For $\varepsilon>0$ and $0<t<t_{0}$

$$
K\left(u_{K} \leqslant \varepsilon\right) \cap H(t) \supset Q(t)\left(u_{Q(t)} \leqslant \varepsilon / 2 t\right) .
$$

Proof. We are going to show that $x \in H(t) \cap K$ implies $u_{K}(x) \leqslant 2 t u_{Q(t)}(x)$. This will prove the lemma.

Notice, first that $M(x)$ lies in the strip $H(a, 0,2 t)$. Then

$$
u(x)=\int_{0}^{2 t} \operatorname{vol}_{d-1}(M(x) \cap H(\tau)) d \tau \leqslant 2 t \operatorname{vol}_{d-1}(M(x) \cap H(t))
$$

because $M(x)$ is centrally symmetric so its largest section is the middle one, $M(x) \cap H(t)$. Next

$$
\begin{aligned}
M(x) \cap H(t) & =\{x+[(K-x) \cap(x-K)]\} \cap H(t) \\
& =x+\{[(K \cap H(t))-x] \cap[x-(K \cap H(t))]\} \\
& =x+[(Q(t)-x) \cap(x-Q(t))] \\
& =M_{Q(t)}(x)
\end{aligned}
$$


Then

$$
u(x) \leqslant 2 t \operatorname{vol}_{d-1} M_{Q(t)}(x)=2 t u_{Q(t)}(x) .
$$

We will now show that for $0<\varepsilon \leqslant 1$

$$
\operatorname{vol} K(u \leqslant \varepsilon) \geqslant \text { const }(d) \varepsilon(\log (1 / \varepsilon))^{d-1} \text {. }
$$

Recalling Lemma 2 this proves that for $\varepsilon \leqslant(2 d)^{-2 d}$

$$
\operatorname{vol} K(v \leqslant \varepsilon) \geqslant \operatorname{vol} K\left(u \leqslant(3 d)^{-e} \varepsilon\right) \geqslant \text { const }(d) \varepsilon(\log (1 / \varepsilon))^{d-1} \text {. }
$$

When $\varepsilon>(2 d)^{-2 d}$, the statement of the theorem follows from the fact that vol $K(v \leqslant \varepsilon)$ is an increasing function of $\varepsilon$.

We prove (7.3) by induction on $d$. The case $d=1$ is trivial. We will need the induction hypothesis in the invariant form (3.6): for $Q \subset R^{d-1}$ compact, convex with vol $d-1, Q>0$ and for $0<\eta \leqslant 1$

$$
\text { vol } Q\left(u_{Q} \leqslant \eta \text { vol } Q\right) / \text { vol } Q \geqslant c_{d-1} \eta(\log (1 / \eta))^{d-2} \text {. }
$$

Assuming this holds we prove (7.3). Write

$$
\begin{aligned}
\operatorname{vol} K(u \leqslant \varepsilon) & =\operatorname{vol}\left[K(u \leqslant \varepsilon) \cap H\left(a, 0, t_{0}\right)\right]=\int_{0}^{t_{0}} \operatorname{vol}_{d-1}[K(u \leqslant \varepsilon) \cap H(t)] d t \\
& \geqslant \int_{0}^{t_{0}} \operatorname{vol}_{d-1} Q(t)\left(u_{Q(t)} \leqslant \varepsilon / 2 t\right) d t
\end{aligned}
$$

according to Lemma 3. Define $\eta=\eta(t)=\varepsilon /(2 t q(t))$ and let $t_{1}$ be the unique solution to $\eta(t)=1$ between 0 and $t_{0}$. Then, by the induction hypothesis, for $t_{1} \leqslant t \leqslant t_{0}$

$$
\begin{aligned}
\operatorname{vol}_{d-1} Q(t)\left(u_{Q(t)} \leqslant \eta q(t)\right) & \geqslant c_{d-1} q(t) \eta(\log (1 / \eta))^{d-2} \\
& =c_{d-1} \frac{\varepsilon}{2 t}\left[\log \left(\frac{2 t q(t)}{\varepsilon}\right)\right]^{d-2} \\
& \geqslant c_{d-1} \frac{\varepsilon}{2 t}\left[\log \left(\frac{2 t}{\varepsilon}\left(\frac{t}{t_{0}}\right)^{d-1} q\left(t_{0}\right)\right)\right]^{d-2},
\end{aligned}
$$

where the last inequality follows from (7.1). We continue (7.4).

$$
\operatorname{vol} K(u \leqslant \varepsilon) \geqslant \int_{t_{t}}^{t_{0}} c_{d-1} \frac{\varepsilon}{2 t}\left[\log \left(\frac{2 t^{d} q\left(t_{0}\right)}{\varepsilon t_{0}^{d-1}}\right)\right]^{d-2} \text {. }
$$

Define $\alpha$ by $\alpha^{d}=2 q\left(t_{0}\right) /\left(\varepsilon t_{0}^{d-1}\right)$ and let $t_{2}=1 / \alpha$. Then, by (7.1) again, $t_{1}<t_{2}<t_{0}$. Substitute now $\tau=\alpha t$ with $\tau_{i}=\alpha t_{i}, i=0,2$. Continue (7.5).

$$
\begin{aligned}
\operatorname{vol} K(u \leqslant \varepsilon) & \geqslant \int_{\tau_{2}}^{\tau_{0}} c_{d-1} \frac{\varepsilon}{2} \frac{1}{\tau}(\log \tau)^{d-2} d \tau=\left.\frac{\varepsilon c_{d-1}}{2(d-1)}(\log \tau)^{d-1}\right|_{\tau=\tau_{2}} ^{\tau=\tau_{0}} \\
& =\frac{\varepsilon c_{d-1}}{2(d-1)}\left[\log \left(\frac{t_{0}\left(2 q\left(t_{0}\right)\right)^{1 / d}}{\left(\varepsilon t_{0}^{d-1}\right)^{1 / d}}\right)\right]^{d-1} \geqslant \frac{\varepsilon c_{d-1}}{2(d-1)}\left(\frac{1}{d} \log \frac{1}{\varepsilon}\right)^{d-1}
\end{aligned}
$$

where the last inequality follows from (7.2). 
§8. Proof of Theorem 3. We prove this theorem for simplices first and then for general polytopes. We may take any simplex $S \subset R^{d}$ because for a nonsingular linear transformation $A$ one clearly has

$$
\operatorname{vol} S\left(v_{S} \leqslant \varepsilon\right) / \operatorname{vol} S=\operatorname{vol} A S\left(v_{A S} \leqslant|\operatorname{det} A| \varepsilon\right) / \operatorname{vol} A S \text {. }
$$

We take a regular simplex $S=\operatorname{conv}\left\{y_{0}, \ldots, y_{d}\right\}$ with vol $S=1$.

Lemma 4. Assume $z \in$ int $S$ and the nearest vertex to $z$ is $y_{0}$. Then

$$
C(z) \supset M\left(\frac{1}{2}\left(z+y_{0}\right)\right) \text {. }
$$

Proof. Let $C(z)=S \cap H(a, t)$ be the minimal cap for $z$. Recall the definition:

$$
H(a, t)=\left\{x \in R^{d}: a . x=h(a)-t\right\} \text { with } h(a)=\max \{a, x: x \in S\} .
$$

We know that $z \in H(a=t)$ and that $z$ is the centre of gravity of the section $S \cap H(a=t)$.

Obviously, $h(a)=a \cdot y_{i}$ for some vertex $y_{i}$. Consider

$$
x \in M\left(\frac{1}{2}\left(z+y_{i}\right)\right)=S \cap\left(z+y_{i}-S\right) .
$$

Then $x=z+y_{i}-y$ with $y \in S$, so

$$
a \cdot x=a \cdot z+a \cdot y_{i}-a \cdot y \geqslant h(a)-t \text {. }
$$

This shows that

$$
M\left(\frac{1}{2}\left(z+y_{i}\right)\right) \subset S \cap H(a, t)=C(z)
$$

for some vertex, $y_{i}$, of $S$.

Assume now that $z$ is closer to $y_{j}$ than to $y_{k}$. We will prove then that $M\left(\frac{1}{2}\left(z+y_{k}\right)\right) \subset C(z)$ does not hold. This will prove the lemma.

Consider the reflection, $z^{\prime}$, of $z$ to the hyperplane bisecting the line segment $\left[y_{j}, y_{k}\right]$. We show that $z^{\prime} \notin C(z)$ and $z^{\prime} \in M\left(\frac{1}{2}\left(z+y_{k}\right)\right)$. By the symmetry of the regular simplex we have $v(z)=v\left(z^{\prime}\right)$. Now $z^{\prime} \in$ int $C(z)$ would imply $v\left(z^{\prime}\right)<v(z)$, a contradiction. And if $z^{\prime}$ were on the bounding hyperplane of $C(z)$, then $C(z)=C\left(z^{\prime}\right)$ must hold. But this cannot be the case because both $z$ and $z^{\prime}$ cannot be the centre of gravity of the section $S \cap H(a=t)$. So $z^{\prime} \notin C(z)$. On the other hand $z^{\prime}=z+\alpha\left(y_{k}-y_{j}\right) \in S$ for some $\alpha \in(0,1)$. Then

$$
z^{\prime}=z+\alpha\left(y_{k}-y_{j}\right)=z+y_{k}-\left[(1-\alpha) y_{k}+\alpha y_{j}\right] \in z+y_{k}-S .
$$

Thus $z^{\prime} \in M\left(\frac{1}{2}\left(z+y_{k}\right)\right)$ and then $M\left(\frac{1}{2}\left(z+y_{k}\right)\right) \subset C(z)$ is indeed impossible.

Define $T_{i}=\left\{x \in S:\left|x-y_{i}\right|=\min \left\{\left|x-y_{j}\right|: j=0, \ldots, d\right\}\right.$. Then

$$
S(v \leqslant \varepsilon)=\bigcup_{i=0}^{d}\left(T_{i} \cap S(v \leqslant \varepsilon)\right) \subset \bigcup_{i=0}^{d}\left\{x \in T_{i}: u\left(\frac{1}{2}\left(x+y_{i}\right)\right) \leqslant \varepsilon\right\},
$$

by Lemma 4 . Thus

$$
\operatorname{vol} S(v \leqslant \varepsilon) \leqslant(d+1) \text { vol }\left\{x \in T_{0}: u\left(\frac{1}{2}\left(x+y_{0}\right)\right) \leqslant \varepsilon\right\} .
$$

Define now an affine transformation $A: R^{d} \rightarrow R^{d}$ with $A y_{0}=0$ and $A y_{i}=e_{i}$ $(i=1, \ldots, d)$ where $e_{1}, \ldots, e_{d}$ form an orthonormal basis of $R^{d}$. Write 
$A x=\left(\xi_{1}, \ldots, \xi_{d}\right)=\xi_{1} e_{1}+\ldots+\xi_{d} e_{d}$. Then $x \in T_{0}, u_{S}\left(\frac{1}{2}\left(x+y_{0}\right)\right) \leqslant \varepsilon$ imply

$$
\xi_{1} \ldots \xi_{d} \leqslant|\operatorname{det} A| \varepsilon \quad \text { and } \quad \max \left\{\xi_{i}: i=1, \ldots, d\right\} \leqslant 1 \text {. }
$$

Similarly as in (8.1) and (3.6)

$$
\frac{\operatorname{vol}\left\{x \in T_{0}: u\left(\frac{1}{2}\left(x+y_{0}\right)\right) \leqslant \varepsilon\right\}}{\operatorname{vol} T_{0}} \leqslant \frac{\operatorname{vol}\left\{\xi \in R^{d}: \xi_{1} \ldots \xi_{d} \leqslant|\operatorname{det} A| \varepsilon, 0 \leqslant \xi_{i} \leqslant 1\right\}}{\operatorname{vol} A T_{0}} .
$$

A simple induction argument shows that for $0<\eta \leqslant 1$

$$
\operatorname{vol}\left\{\xi \in R^{d}: \xi_{1} \ldots \xi_{d} \leqslant \eta, 0<\xi_{i}<1\right\}=\eta \sum_{j=0}^{d-1} \frac{1}{j !}\left(\log \frac{1}{\eta}\right)^{j} .
$$

But det $A$ is a constant depending on $d$ only so for $\varepsilon<\varepsilon_{0}(d)$ we get

$$
\text { vol } S(v \leqslant \varepsilon) \leqslant(d+1) \text { vol }\left(T_{0} \cap S(v \leqslant \varepsilon)\right) \leqslant \text { const }(d) \varepsilon(\log (1 / \varepsilon))^{d-1} \text {. }
$$

Now we prove the theorem for general polytopes $P \subset R^{d}$. Take a triangulation of $P$ into simplices $S_{1}, \ldots, S_{m}$ using vertices of $P$ only. Then

$$
P\left(v_{P} \leqslant \varepsilon\right) \subset \bigcup_{i=1}^{m} S_{i}\left(v_{S_{i}} \leqslant \varepsilon\right) .
$$

With suitable (nonsingular) affine transformations $A_{i}: R^{d} \rightarrow R^{d}$ such that $A_{i} S_{i}=S$ we have

$$
\begin{aligned}
\operatorname{vol} P\left(v_{P} \leqslant \varepsilon\right) & \leqslant \sum_{i=1}^{m} \operatorname{vol} S_{i}\left(v_{S_{i}} \leqslant \varepsilon\right)=\sum_{i=1}^{m} \frac{\operatorname{vol} S_{i}}{\operatorname{vol} A S_{i}} \operatorname{vol} A S_{i}\left(v_{A S_{i}} \leqslant\left|\operatorname{det} A_{i}\right| \varepsilon\right) \\
& \leqslant \varepsilon \sum_{i=1}^{m}\left(\log \left(1 /\left(\left|\operatorname{det} A_{i}\right| \varepsilon\right)\right)\right)^{d-1} \leqslant \operatorname{const}(P) \varepsilon\left(\log \frac{1}{\varepsilon}\right)^{d-1}
\end{aligned}
$$

We mention here that there is an alternative proof for this theorem using the arguments of the proof of Theorem 2.

\$9. Proof of Theorem 4. It is clear that for some $\rho>0, \delta>0$ the set of $(\rho, \delta)$-circular points $\Omega$ form a set of positive measure in $\partial K$. Take $p \in \Omega$ and consider $z=p-\alpha q$ where $q$ is the outer unit normal to $K$ at $P$. Assume

$$
\alpha \leqslant \min \left(\frac{\delta}{d}, \varepsilon^{2 /(d+1)} \rho^{-(d-1) /(d+1)} \omega_{d-1}^{-(d-1) /(d+1)}\right) .
$$

Claim 2. $z \in K(v \leqslant \varepsilon)$.

Proof. Assume this is false. Then for a minimal cap $C(z)=K \cap H(a, t)$ one has vol $C(z)>\varepsilon$. Take a chord $[x, y]$ through $z$ of $K$ lying in the bounding hyperplane $H(a=t)$ of $C(z)$. Consider a minimal cap $C^{\prime}(z)$ of the ball $B^{\rho}=B(\rho, p-\rho a)$. As $p$ is $\rho$-circular, one of the endpoints of the chord $[x, y]$, $x$ (say), lies in the cap $C^{\prime}(z)$. But $z$ is the centre of gravity of the section $K \cap H(a=t)$ and so, according to a well-known result (see [7], e.g.)

$$
(d-1)|z-x| \geqslant|y-z| \text {. }
$$


CONVEX BODIES, ECONOMIC CAP COVERINGS, RANDOM POLYTOPES 289

This shows that $y$ lies in the minimal cap $C^{\prime}\left(z^{\prime}\right)$ of $B^{\rho}$ where $z^{\prime}=p-d \alpha a$. As $y$ is an arbitrary point of the section $K \cap H(a=t)$, we have that $C(z) \subset C^{\prime}\left(z^{\prime}\right)$. Then

$$
\text { vol } C(z) \leqslant \operatorname{vol} C^{\prime}\left(z^{\prime}\right) \leqslant(\alpha d)^{(d+1) / 2}(2 \rho)^{(d-1) / 2} \omega_{d-1} .
$$

By the choice of $\alpha$ this is less than $\varepsilon$. A contradiction.

We claim now that

$$
\operatorname{vol} K(v \leqslant \varepsilon) \geqslant \text { const }(K) \alpha \operatorname{vol}_{d-1} \Omega \text {. }
$$

This will prove the theorem for $\alpha=$ const $\varepsilon^{2 /(d+1)}$ if $\varepsilon$ is small enough. Define first

$$
L_{s}=\{p \in \delta K: K \supset B(s, p-s a)\} .
$$

It is well-known [27] that $\operatorname{vol}_{d-1}\left(\delta K \backslash L_{s}\right) \rightarrow 0$ as $s \rightarrow 0$. Choose $s>0$ so that $\operatorname{vol}_{d-1}\left(\Omega \cap L_{s}\right) \geqslant \frac{1}{2} \operatorname{vol}_{d-1} \Omega$. Now to see (9.1) we use the proof of the cap covering theorem (Theorem 6). So choose a maximal system of points $x_{1}, \ldots, x_{m}$ from $\delta K(v \geqslant \varepsilon)$ subject to the conditions:

$$
M\left(x_{i}, \frac{1}{2}\right) \cap M\left(x_{j}, \frac{1}{2}\right)=\varnothing ;
$$

the centre of the minimal cap $C\left(x_{i}\right)$ lies in $\Omega \cap L_{s}$.

So let $C\left(x_{i}\right)=K \cap H\left(a_{i}, t_{i}\right)$ with centre $p_{i} \in \Omega \cap L_{s}$. We know from the proof of the cap covering theorem that

$$
\Omega \cap L_{s} \subset \bigcup_{i=1}^{m} M\left(x_{i}, 5\right) \subset \bigcup_{i=1}^{m} K_{i}
$$

where $K_{i}=K \cap H\left(a_{i}, 6 t_{i}\right)$. According to Claim 2, the width of the cap $C\left(x_{i}\right)$ is at least $\alpha$, so the width of $K_{i}$ is at least $6 \alpha$. Then

$$
\begin{aligned}
\operatorname{vol} K(v \leqslant \varepsilon) & \geqslant \sum_{i=1}^{m} \frac{1}{2} \operatorname{vol} M\left(x_{i}, \frac{1}{2}\right) \\
& \geqslant \operatorname{const}(d) \sum_{i=1}^{m} \operatorname{vol} K_{t} \\
& \geqslant \text { const }(d) \frac{6 \alpha}{d} \sum_{i=1}^{m} \operatorname{vol}_{d-1}\left(K \cap H\left(a_{i}=6 t_{i}\right)\right) .
\end{aligned}
$$

Now vol $\operatorname{di-1}\left(K \cap H\left(a_{i}=6 t_{i}\right)\right) \geqslant \mathrm{const}(d, \rho, s) \operatorname{vol}_{d-1}\left(\partial K \cap K_{i}\right)$. This follows from the fact that the outer normals to $K$ at the points of $\partial K \cap K_{i}$ cannot differ much from $a_{i}$ (if $\varepsilon$ is small enough) because $p_{i}$ is in $L_{s}$. Using this we get

$$
\begin{aligned}
\operatorname{vol} K(v \leqslant \varepsilon) & \geqslant \text { const }(d, \rho, s) \alpha \sum_{i=1}^{m} \operatorname{vol}_{d-1}\left(\partial K \cap K_{i}\right) \\
& \geqslant \operatorname{const}(K) \alpha \operatorname{vol}_{d-1}\left(\Omega \cap L_{s}\right) \geqslant \operatorname{const}(K) \frac{1}{2} \alpha \operatorname{vol}_{d-1}(\Omega) .
\end{aligned}
$$

\$10. Proof of Theorem 8. Let $P \subset R^{d}$ be a convex polytope having $n$ vertices and assume vol $P=1$. Set

$$
\varepsilon=c_{0}(d) n^{-(d+1) /(d-1)}
$$

where the constant $c_{0}(d)$ is to be determined later. 
We assume that $n$ is large enough to ensure that $\varepsilon<(2 d)^{-2 d}$. Then Theorem 6 applies: there are caps $K_{1}, \ldots, K_{m}$ and subsets $K_{1}^{\prime}, \ldots, K_{m}^{\prime}$ satisfying (i), (ii) and (iii) of Theorem 6. Then

$$
\begin{aligned}
m(6 d)^{-d} \varepsilon & \leqslant \sum_{i=1}^{m} \operatorname{vol} K_{i}^{\prime} \leqslant \operatorname{vol} P(v \leqslant \varepsilon) \\
& \leqslant 4 E(P,\lceil 1 / \varepsilon\rceil) \leqslant 4 c(d)(\lceil 1 / \varepsilon\rceil)^{-2 /(d+1)} \leqslant 8 c(d) \varepsilon^{2 /(d+1)}
\end{aligned}
$$

where the third inequality follows from Theorem 1 and the fourth from (1.5) and (1.4) with a suitable constant $c(d)$. This shows that

$$
m \leqslant 8(6 d)^{d} c(d) \varepsilon^{-(d-1) /(d+1)} \leqslant 8(6 d)^{d} c(d) c_{0}(d)^{-(d-1) /(d+1)} n \leqslant \frac{1}{d+1} n
$$

if we choose $c_{0}(d)$ large enough.

Now the caps $K_{1}, \ldots, K_{m}$ cover $P(v \leqslant \varepsilon)$ so they cover the boundary of $P$ as well. Then there is a cap, $K_{i}$ say, containing at least $n / m \geqslant d+1$ vertices, $y_{0}, \ldots, y_{d}$ of $P$. Consequently conv $\left\{y_{0}, \ldots, y_{d}\right\} \subset K$ and

$$
\text { vol conv }\left\{y_{0}, \ldots, y_{d}\right\} \leqslant \operatorname{vol} K_{i} \leqslant 6^{d} \varepsilon \leqslant \text { const }(d) n^{-(d+1) /(d-1)} .
$$

\$11. Proof of Theorem 9. Lemma 1 implies $K(v \leqslant \varepsilon) \subset K(u \leqslant 2 \varepsilon)$. By Lemma 2, if $\varepsilon \leqslant \varepsilon_{1}(d)=\frac{1}{2}\left(12 d^{3}\right)^{-d}$, then $K(u \leqslant 2 \varepsilon) \leqslant K\left(v \leqslant 2(3 d)^{d} \varepsilon\right)$ so indeed

$$
K(v \leqslant \varepsilon) \subset K(u \leqslant 2 \varepsilon) \subset K\left(v \leqslant 2(3 d)^{d} \varepsilon\right) .
$$

Computing volumes here and applying Theorem 7 gives

$$
\operatorname{vol} K(v \leqslant \varepsilon) \leqslant \operatorname{vol} K(u \leqslant 2 \varepsilon) \leqslant c_{1}(d) \text { vol } K(v \leqslant \varepsilon) .
$$

Acknowledgment. The authors are grateful to Professor Ravi Kannan for several inspiring discussions.

\section{References}

1. V. I. Arnold. Statistics of integral convex polytopes. Functional Analysis and its Appl., 14 (1980), 1-3.

2. I. Bárány and Z. Füredi. On the shape of the convex hull of random points. Probab. Th. Rel. Fields, 77 (1988), 231-240.

3. W. Blaschke. Vorlesungen uber Differentialgeometrie II. Affine Differentialgeometrie (Berlin, Springer, 1923).

4. C. Buchta. Stochastische Approximation konvexer Polygone. Z. Wahrscheinlichkeitstheorie verw. Gebiete, 67 (1984), 283-304.

5. C. Buchta. Zufallige Polieder, eine Obersicht, Lecture Notes in Mathematics 1114 (Berlin, Springer, 1985), 1-13.

6. C. Buchta. Private communication, 1987.

7. L. Danzer, B. Grunbaum and V. Klee. Helly's theorem and its relatives. Proc. Symp. Pure Math., vol. VIII, Convexity (AMS, Providence, RI, 1963).

8. R. A. Dwyer, On the convex hull of random points in a polytope. To appear in J. Applied Prob.

9. G. Ewald, L. G. Larman and C. A. Rogers. The directions of the line segments and of the $r$-dimensional balls on the boundary of a convex body in Euclidean space. Mathematika, 17 (1970), 1-20.

10. Z. Füredi. Random polytopes in the $d$-dimensional cube. Discrete and Comp. Geometry, 1 (1986), 315-319. 
11. H. Groemer. On the mean value of the volume of a random polytope in a convex set. Arch. Math., 25 (1974), 86-90.

12. P. M. Gruber. Approximation of convex bodies, Convexity and its applications, ed. P. M. Gruber and J. M. Wills (Basel, Birkhauser, 1983).

13. P. M. Gruber. In most cases approximation is irregular. Rendiconti Sem. Mat. Torino, 41 (1983), 19-33.

14. R. Kannan. Private communication, 1987.

15. J. Komlós, J. Pintz and E. Szemerédi. A lower bound for Heilbronn's problem. J. London Math. Soc. (2), 25 (1982), 13-24.

16. S. B. Konyagin and K. A. Sevastyanov. Estimation of the number of vertices of a convex integral polyhedron in terms of its volume. Functional Analysis and its Appl., 18 (1984), 13-15.

17. A. M. Macbeath. A theorem on non-homogeneous lattices. Annals of Math. (2), 56 (1952), 269-293.

18. A. Rényi and R. Sulanke. Uber die konvexe Hulle von $n$ zufallig gewahlten Punkten. Z. Wahrscheinlichkeitstheorie verw. Gebiete, 2 (1963), 75-84.

19. A. Rényi and R. Sulanke. Uber die konvexe Hulle von $n$ zufallig gewahlten Punkten II. Z. Wahrscheinlichkeitstheorie verw. Gebiete, 3 (1963), $138-184$.

20. K. F. Roth. On a problem of Heilbronn, III. Proc. London Math. Soc. (3), 25 (1972), 543-549.

21. R. Schneider and J. A. Wieacker. Random polytopes in a convex body. Z. Wahrscheinlichkeitstheorie verw. Gebiete, 52 (1980), 69-73.

22. R. Schneider. Approximation of convex bodies by random polytopes. Aequationes Math., 32 (1987), 304-310.

23. J. G. Wendel. A problem in geometric probability. Math. Scand., 11 (1962), 109-111.

24. J. A. Wieacker. Einige Probleme der polyedrischen Approximation. Diplomarbeit (Frieburg i. Br., 1987).

25. G. E. Andrews. A lower bound for the volumes of strictly convex bodies with many boundary points. Trans. Amer. Math. Soc., 106 (1965), 270-279.

26. K. Leichtweiss. Uber eine Formel Blaschkes zur Affinoberfläche. Studia Math. Hung., 21 (1986), 453-474.

27. R. Schneider. Boundary structure and curvature of convex bodies. Proc. Geom. Symp., Siegen, 1978 (Birkhäuser, Basel, 1979), 13-59.

Dr. I. Bárány,

The Mathematical Institute of the Hungarian Academy of Sciences,

1365 Budapest,

P.O.B. 127 ,

Hungary

Professor D. G. Larman,

Department of Mathematics,

University College London,

Gower Street,

London. WC1E 6BT
52A22: CONVEX SETS AND RELATED GEOMETRIC TOPICS; Random convex sets and integral geometry.

Received on the 9 th of March, 1988. 